\title{
Altmetrics: medición de la influencia de los medios en el impacto social de la investigación
}

\author{
Julio Alonso Arévalo \\ José Antonio Cordón-Garcia \\ Bruno Maltrás Barba
}

Recibido: 25 de mayo de 2016

\section{Resumen:}

Los medios sociales están cambiando la forma de interactuar, presentar las ideas e información y juzgar la calidad de los contenidos y contribuciones. En los últimos años han surgido cientos de plataformas que permiten compartir libremente todo tipo de información y conectarnos a través de redes. Estas nuevas herramientas generan estadísticas de actividad e interacciones entre sus usuarios tales como menciones, retweets, conversaciones, comentarios en Blogs o en Facebook; gestores de referencias que muestran índices de popularidad de las referencias más compartidas por otros investigadores o repositorios que generan estadísticas de visitas o descargas de artículos. En este documento se analiza que implicaciones y significado tiene altmetrics, cuáles son sus ventajas y críticas, plataformas (Almetric.com, ImpactStory, Plos Altmetrics, PlumX), avances y que beneficios reporta para autores, editores y bibliotecarios. Concluyendo que el valor de las métricas alternativas como una herramienta complementaria de análisis de citas es evidente, aunque se sugiere que se debe investigar más profundamente sobre esta cuestión para desentrañar el significado y el valor potencial de estos indicadores para la evaluación de todo su potencial.

\section{Palabras clave:}

Palabras clave: Altmetrics, impacto, Evaluación, Calidad, Investigación, Redes sociales, Bibliometria 


\section{Almetrics: measuring the influence of the media on the social impact of research}

\section{Abstract:}

Social media is changing the way we interact, present ideas and information and judge the quality of content and contributions. In recent years there have been hundreds of platforms to freely share all kinds of information and connect across networks. These new tools generate activity statistics and interactions among users such as mentions, retweets, conversations, comments on blogs or Facebook; managers references showing popularity ratings of more references shared by other researchers or repositories that generate statistics of visits or downloads of articles. This paper analyzes that have meaning and implications altmetrics, what are its advantages and critical platforms (Almetric.com, ImpactStory, Plos altmetrics, PlumX), reports progress and benefits for authors, publishers and librarians. It concluded that the value of alternative metrics as a complementary tool citation analysis is evident, although it is suggested that you should dig deeper into this issue to unravel the meaning and the potential value of these indicators to assess their potential.

\section{Keywords:}

Altmetrics, impact, Evaluation, Quality, Research, Social Networks, bibliometry

\section{Introducción}

Las instituciones, así como los investigadores que trabajan en ellas, necesitan sistemas que midan la calidad de sus intervenciones, que ponderen las buenas prácticas y que sirvan como referentes comparativos para estudios de proyección y visibilidad de las mismas. Estas mediciones se han basado fundamentalmente en criterios de carácter cuantitativo, tomando indicadores como el factor de impacto de las publicaciones o el índice $\mathrm{H}$, como referentes incontrovertibles de la medición. La cuestión es que al hilo de las innovaciones que la red ha introducido en los procesos de comunicación científica y las posibilidades que estas ofrecen, es preciso replantear igualmente la incidencia de los sistemas de medición de la ciencia tomando en consideración parámetros complementarios o alternativos. 
En 2015 se publicó el manifiesto de Leiden sobre Indicadores de Investigación (Hicks et al., 2015). En este manifiesto se deja constancia de que el problema de la evaluación de la investigación es que pasó de estar basada en valoraciones de expertos a depender de métricas estrictamente cuantitativas. Hicks y Wouters señalan como la obsesión por el factor de impacto es un fenómeno relativamente nuevo, cuyo mal uso ha distorsionado el panorama académico por un exceso de incentivación de la publicación de artículos frente a otras valiosas contribuciones al conocimiento.

Wouters y Rijcke (2014) habían establecido el concepto de Evaluation Gap contraponiendo la brecha existente entre los controles de calidad de las publicaciones científicas y las nuevas funciones de la investigación en la sociedad. Los autores recomiendan repensar los sistemas de evaluación teniendo en cuenta tanto los procesos cuantitativos, como aquellos otros que sirvan para contextualizar aquella mediante procedimientos de carácter cualitativo. El problema, comentan, surge cuando la evaluación pasa de ser un medio a erigirse un fin en sí misma. Como define la Ley de Goodhart cuando una medida se convierte en un objetivo deja ser una buena medida.

Por otra parte, la comunicación científica, gracias a las posibilidades de la red y de la edición digital, se está diversificando por senderos alternativos, cada vez más frecuentados por el autor, cuando considera la formulación de sus hipótesis o la presentación de sus primeros resultados de investigación, pero también cuando se plantea la valoración de sus contribuciones por parte de la comunidad científica. Blog, microblog, redes sociales, comunidades virtuales, constituyen espacios emergentes de intervención académica que escapan a los rígidos protocolos de las publicaciones científicas convencionales. Las páginas de grupos de investigación en Facebook, su presencia en Twitter, las redes surgidas al albor de Mendeley, etc., están configurando un espacio de intercambio, colaboración y transmisión de información original cada vez más influyente. Cualquier autor se ha convertido en potencialmente hipervisible, constructor de su reputación virtual, curador de los contenidos que genera y regulador de tráfico hacia ellos o hacia los colegas.

La comunicación científica se plasma en un elenco de actividades cada vez más amplia y diversificada, entre las que la publicación convencional (artículos, capítulos de libros, 
libros, comunicaciones, etc.) constituye un elemento relevante pero no único, y en que la medición de su repercusión ya no puede quedar circunscrita exclusivamente a la cita. La presencia de una obra en bibliografías recomendadas ¿constituye una evidencia sólida de su calidad? ¿Las descargas que un artículo recibe en una red social de investigación como Research Gate o Academia.edu pueden ser un referente de la misma? Las ventas de un libro, como propone Expósito (2016), pueden constituir un indicador de relevancia si se ponen en relación con las tiradas y las áreas de conocimiento en la que se inscriben iy las ediciones de una obra? O los préstamos en bibliotecas, etc.

Es por esto que en, prácticamente todos los ámbitos de investigación, las herramientas digitales se han convertido en indispensables, la aparición de nuevos paradigmas cómo el acceso abierto, big data, datos enlazados, métricas alternativas y redes sociales son un ejemplo importante de cómo estos cambios están afectando a la forma en que los investigadores piensan en el futuro de las publicaciones académicas. Servicios basados en la Web de todo tipo producen enormes cantidades de datos como resultado de su uso, tanto de la actividad académica como pública. Hace unos meses, la Universidad de Utrech llevó a cabo una encuesta sobre las herramientas más populares utilizadas por los investigadores de todo el mundo "Most popular tools for single research activities" (Innovations, 2015). Los resultados son sumamente significativos, por cuanto muestran que herramientas están utilizando los investigadores, cuáles son las emergentes, y cuales tienen una mayor proyección de futuro. A la pregunta de qué sitios web utilizan para buscar literatura profesional ponen de manifiesto que las herramientas más utilizadas son Google Schoolar en un $92 \%$ de los casos, seguido de Web of Science en un $47 \%$, PubMed un $45 \%$, Scoupus un $29 \%$, Mendeley un $22 \%$ y WorldCat en en $20 \%$.

La mayoría de los investigadores recurren al repositorio de su propia institución para depositar y dar visibilidad a sus trabajos de investigación en un $53 \%$ de las respuestas. Otro sitio popular para depositar los trabajos de investigación es ResearchGate (50\%) en el que difunden sus investigaciones prácticamente la mitad de los autores. De los repositorios temáticos los más populares y utilizados son PubMed y ArXiv con un 18 y un $19 \%$ respectivamente. En el ámbito de las Ciencias Sociales SSRN (5\%). Además la mitad de los investigadores utilizan Web of Science para medir el impacto de su investigación, Journal Citation Report de Thompson es utilizado por el 45\%, y Almetric.com (44\%) se 
configura como la tercera herramienta utilizada para este fin, incluso por delante de Scopus, y otros recursos altmétricos como ImpactStory o Ploos article level metrics.

Es el caso de herramientas como Mendeley, un gestor de referencias social nacido en el año 2008 con más de 4 millones de usuarios y más de 120 millones de referencias compartidas, cuando bases de datos nacidas en los años 60 como la actual ISI Web of Knowledge se calcula que tiene en torno a 57 millones de referencias. La importancia de este gestor va más allá de lo puramente instrumental, ya que lo más importante de esta herramienta es que ofrece datos sobre el comportamiento de los investigadores. Estos datos proporcionados por Mendeley -que Elsevier adquirió en 2013-, proporcionan una fuente invaluable y libre sobre situación de los investigadores en todas las disciplinas.

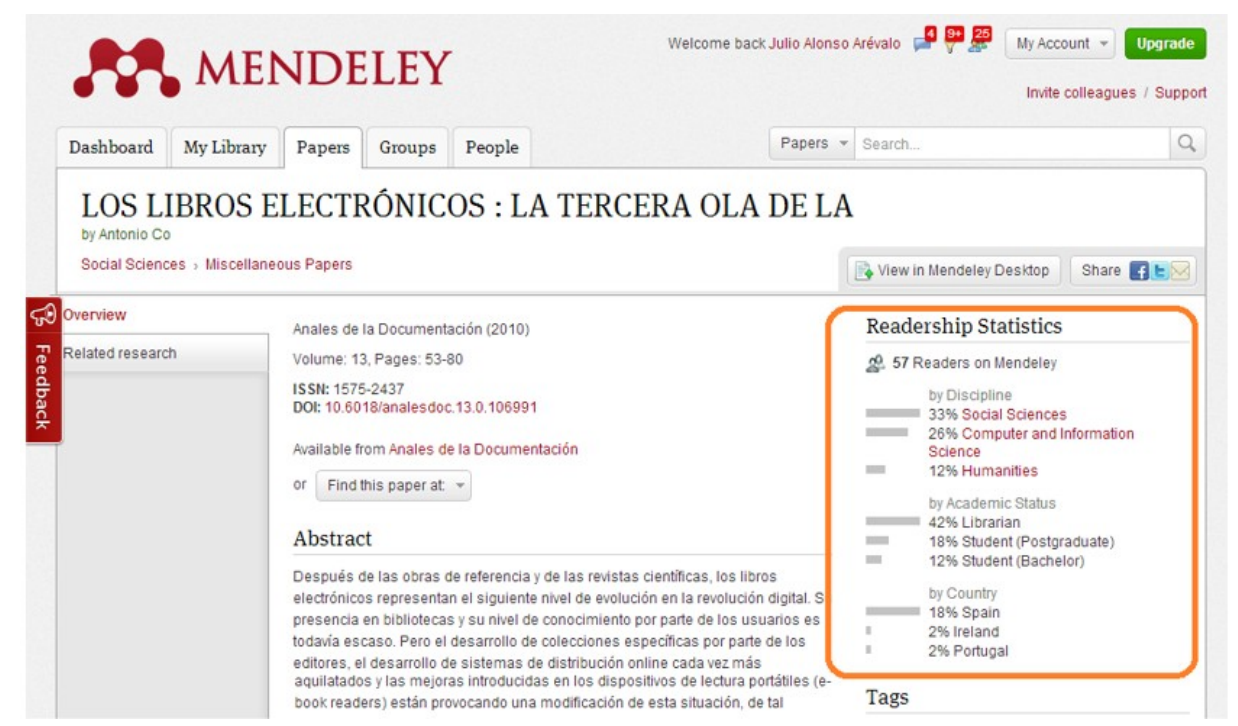

Fig.1. Datos de uso de un registro de Mendeley

En otra investigación, publicada en marzo de 2016, sobre sistemas de descubrimiento en publicaciones científicas (Gardner and Inger, 2016) se pueden apreciar las modificaciones operadas en las prácticas de acceso y búsqueda de la información científica en una amplia comunidad de usuarios. La investigación se ha realizado a partir de 40.000 respuestas, recibidas de una amplia comunidad de usuarios procedentes de sectores científicos y académicos diferentes.

A lo largo de todo el estudio se puede apreciar la importancia que cobran las nuevas herramientas de descubrimiento científico en las prácticas de consumo de los 
investigadores. Es muy significativo el cambio operado a lo largo de los últimos diez años cuando se analizan los datos desde una perspectiva diacrónica. Un cambio que permite comprobar la consolidación de sistemas emergentes como alternativa a las vías más tradicionales.

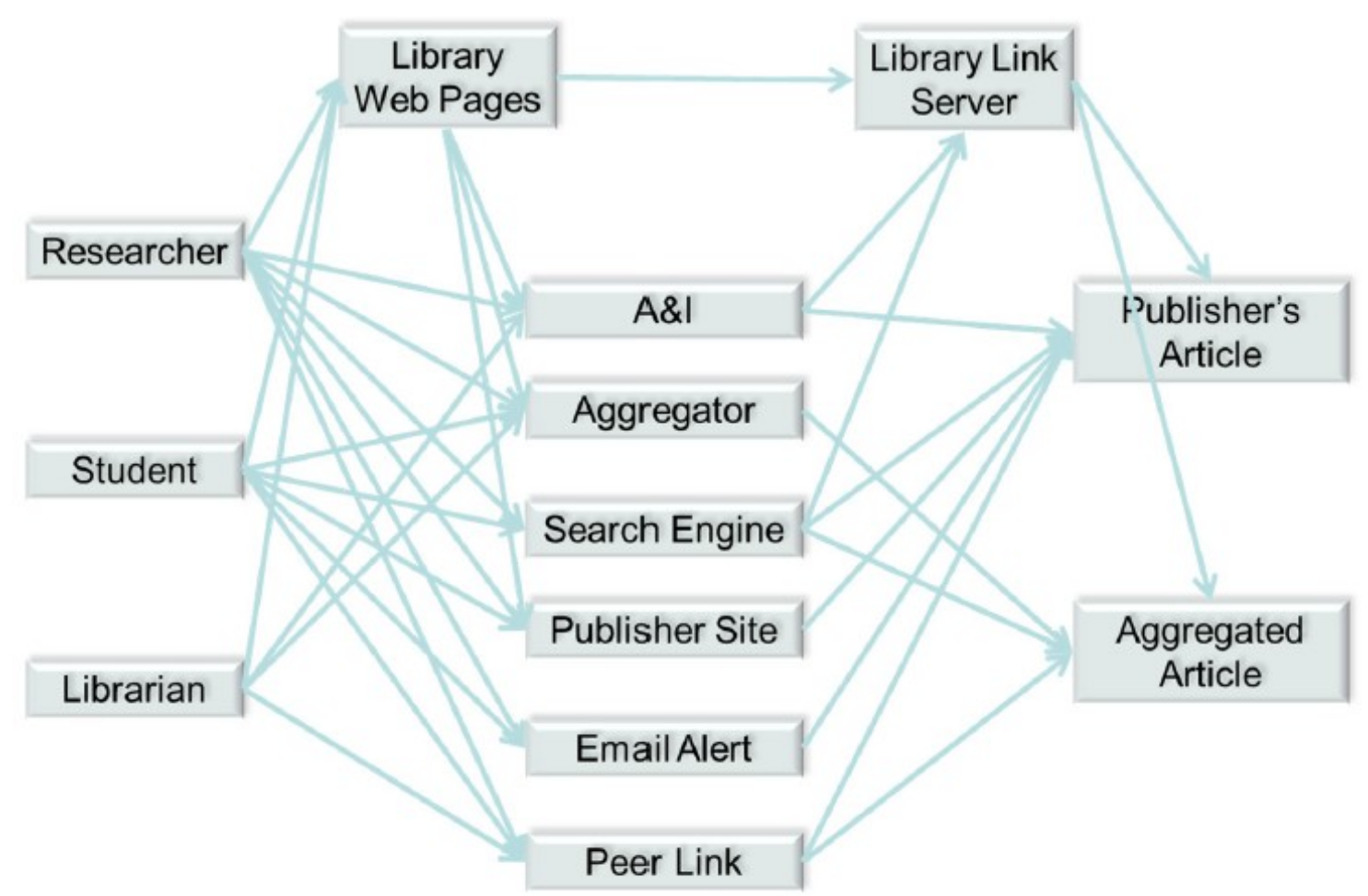

Fig. 2. Relaciones y medición de sistemas de descubrimiento. Fuente Gardner and Inger (2016)

Las interrelaciones múltiples que se establecen entre las diferencias instancias que intervienen en la investigación dan lugar a sistemas de medición que permiten relacionar contextos que habitualmente quedaban fuera de los procesos valorativos.

Uno de los resultados más interesante del estudio es la creciente importancia de las redes sociales en los sistemas de reconocimiento y descubrimiento de artículos, en todas las áreas de conocimiento 
When you need to do a search for articles on a specific subject, where on the web do you start that search? Importance of Social Media in Academic Sector by Subject

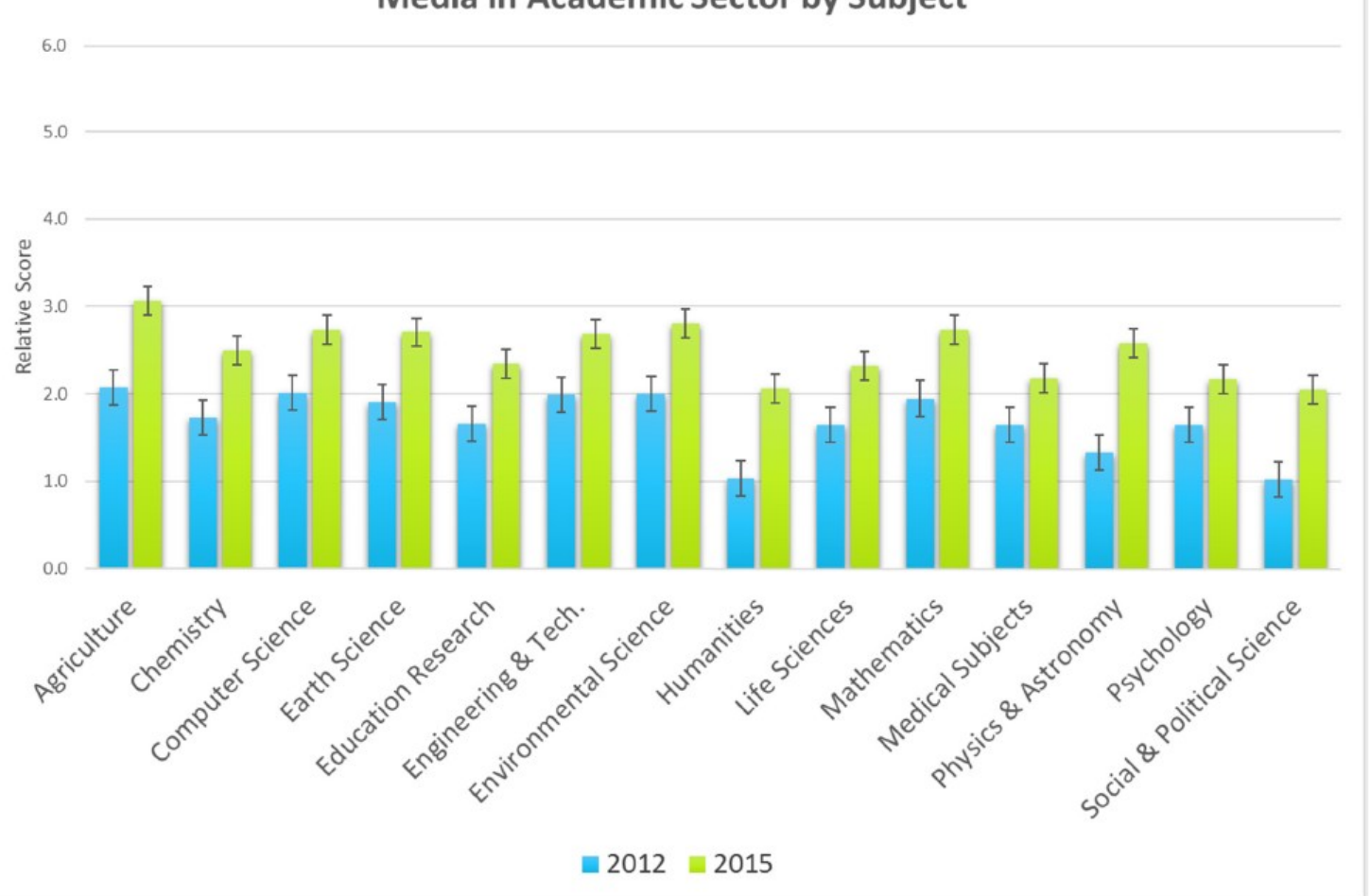

Fig. 3. Influencia de las redes sociales en la búsqueda y descubrimiento de artículos. Fuente Gardner and Inger (2016)

En los últimos años hemos asistido a un avance asombroso en el desarrollo de sistemas de identificación de publicaciones y de autores, herramientas absolutamente esenciales para la interconexión de sistemas científicos de cara a la búsqueda y recuperación de información en las diferentes bases de datos. Es el caso de ORCID, un proyecto abierto, sin ánimo de lucro, comunitario, que ofrece un sistema para la identificación inequívoca de investigadores y un método universal para vincular las actividades de investigación y los productos de estos identificadores como DOI o ORCID. ORCID integra otros sistemas de identificación precedente como ISNI, VIAF, NAF, Research ID,.. etc. y es capaz de interactuar con las principales bases de datos como Scopus, CrossRef, Readlyc y otras. Estos identificadores cobran aún más importancia cuando hablamos de métricas a nivel de autor (Altmetrics), ya que recopilan datos a través de plataformas tales como Altmetric.com, Plum Analytics e ImpactStory.org, o de redes sociales académicas como ResearchGate.net, Academia.edu, GetCited.org. Fuentes todas ellas de importancia 
capital cuando se trata de evaluar la actividad de autores e investigadores de artículos en redes sociales académicas, redes generales, o revistas con indicadores bibliométricos. Estos acontecimientos están haciendo replantearse nuevas posibilidades y desafíos en la evaluación de la calidad de la investigación, también a nivel de investigadores individuales, organismos de financiación y desarrollos de carrera.

Un entorno digital en expansión impulsa cambios en los criterios para medir el impacto de la investigación y la erudición. Muchos de estos trabajos de investigación se conocen o se publica en la web, por lo que es importante disponer de un método para el seguimiento del impacto de un trabajo en estos nuevos medios de comunicación. Altmetrics, abreviatura de métricas alternativas, es una metodología de desarrollo rápido para medir el impacto de los trabajos académicos y de investigación publicados en el web. Los defensores de Altmetrics sostienen que la utilización de las citas de artículos y los factores de impacto de las revistas como medios exclusivos para la evaluación de la investigación no miden con suficiente precisión el impacto de los artículos basados en la web o la comunicación académica entre los científicos, académicos e investigadores. Altmetrics, integra todas las métricas, no excluye, si no que complementa a los medios tradicionales que miden el impacto académico y el proceso de revisión por pares. Estas métricas son cada vez más importantes para conocer como los investigadores utilizan los programas de Internet para organizar y compartir sus artículos con otros colegas a través de herramientas como Mendeley, Figshare Impact Story, y PLOS, OA, o sitios de redes sociales científicas o generales para compartir artículos científicos tales como Academia.edu y ResearchGate. Fuentes todas ellas de importancia capital cuando se trata de evaluar la actividad de autores e investigadores de artículos a nivel de métricas (Altmetrics), redes sociales académicas redes o revistas regionales con indicadores bibliométricos (Das, 2015).

Por otra parte con la llegada del siglo XXI se empiezan a consideran necesarias nuevas metodologías para medir la ciencia. Organismos públicos y otras entidades han empezado a cuestionarse esta forma de medición científica. Así en el año 2012 se hizo pública una declaración mundial que abarca a todas las disciplinas académicas, la llamada "Declaración de San Francisco sobre Evaluación de la Investigación” (DORA), fue impulsada por la Sociedad Americana de Biología Celular (BCSV), junto con un grupo de 
directores y editores de revistas científicas, y reconocía la necesidad de mejorar la forma en que se evalúan los resultados de la investigación científica. Esta declaración se basa en tres recomendaciones: 1. La necesidad de eliminar el uso de métricas basadas en revistas, tales como índice de impacto de revistas, en la financiación, en los nombramientos, y en las consideraciones de promoción;2. La necesidad de evaluar la investigación por sus propios méritos y no en base a la revista en la que se publica la investigación, y 3. La necesidad de aprovechar las oportunidades que ofrece la publicación en línea (como relajar los límites innecesarios en el número de palabras, figuras y referencias en artículos, y la exploración de nuevos indicadores de la importancia y el impacto). En este mismo sentido se ha pronunciado el International Council of Science que dice literalmente:

"Quienes participan en la administración de la investigación utilizan métricas tradicionales para la evaluación de la importancia y el impacto de la investigación Estas métricas a su vez afectan el comportamiento de investigadores, tales como la elección de revistas, ya que se busca maximizar su desempeño, como por las métricas utilizadas, lo que contribuye al mantenimiento de los altos precios de las publicaciones. La apertura y el compartir, permite un nuevo reconocimiento del impacto de la investigación a través de nuevas contribuciones y la generación de conjuntos de datos, software, código, blogs, wikis y foros. Las métricas utilizadas en la evaluación de la investigación y los investigadores debieran ayudar a promover el acceso abierto y la ciencia abierta, y la comunidad científica debe participar plenamente en su diseño" (Science, 2014)

\section{¿Qué es Altmetrics?}

La influencia generada por los medios sociales ha sido calificada como Investigación 2.0, Social Reference, Altmetrics, (Priem, Taraborelli y Groth 2010). Varios autores han investigado la influencia en Twitter, en Blogs y gestores de referencias. Sin lugar a dudas la mayor parte de los investigadores han trasladado sus actividades de investigación a la web y con el éxito de los medios sociales esta situación se ha hecho más evidente, ya que estas herramientas tienen más potencialidad para desarrollar un rango mayor de influencia académica que los entornos tradicionales de publicación (Priem, Piwowar y Hemminger, 2011). A pesar de que aún las métricas tradicionales basadas en el impacto de la publicación y en las citas recibidas siguen teniendo una fuerte influencia en la comunidad académica, los medios sociales como blogs, repositorios, redes sociales y 
gestores de referencias en línea están empezando a ser considerados con el objetivo de obtener una imagen más completa acerca del impacto de las publicaciones. Sitios como Google Schoolar incluyen muchos tipos de publicaciones como preprints, presentaciones, artículos o tesis, que no aparecen en los índices de citas tradicionales como Web of Science o Scopus, y que de alguna manera reflejarían una más amplia tipología de impacto (Aguillo, 2011).

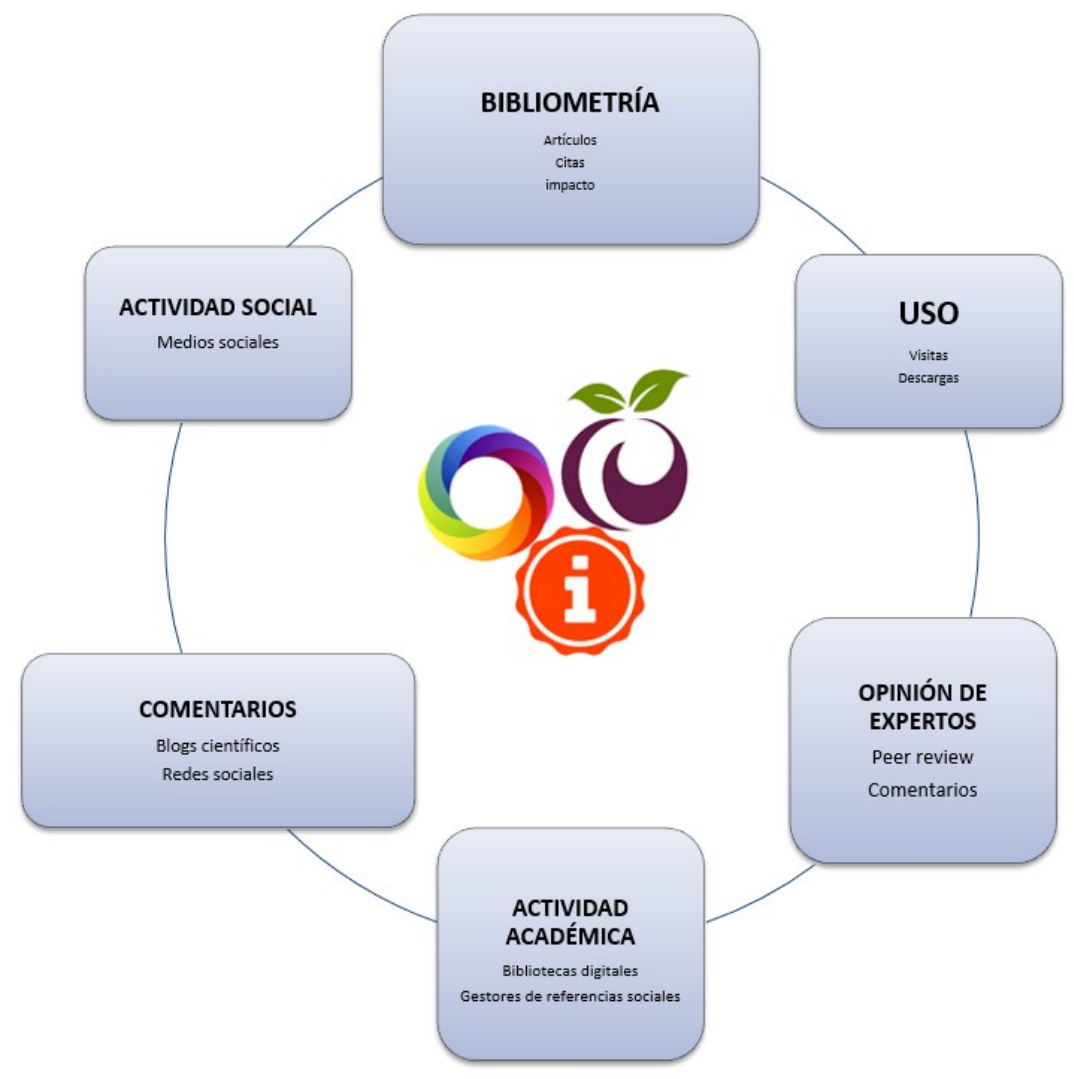

Fig.4 . Altmetrics

Bibliotecas digitales, repositorios OA, revistas y bases de datos de artículos permiten descargar un documento y disponer de estadísticas de uso; éstas tienen un importante potencial para establecer la popularidad de un artículo, y su potencial de lectura, lo que de alguna manera es un buen indicador de su valor o influencia científica. Algunos estudios también han hecho la correlación entre el número de vistas y descargas a un documento y el número de citas recibidas, lo que sugiere de alguna manera que las estadísticas de uso tienen una relación directa con su impacto; aunque también existen algunos problemas prácticos respecto al uso de estas estadísticas para la evaluación de 
la investigación, como la falta de uniformidad y normalización, o que los propios editores las manipulen para obtener una mayor influencia. Aunque las mayores críticas se centran en decir que se trata de una simple medida de uso y no de influencia científica (Neylon, 2011). Una serie de investigadores han trabajado en la identificación de nuevos métodos cuantitativos de evaluación de la investigación para la Web con el objetivos de complementar el análisis de citas tradicional (Burgelman, Osimo y Bogdanowicz, 2010) (Thelwall et al., 2013) (Thelwall, Haustein, Larivière, \& Sugimoto, 2013). A este respecto se pueden diferenciar dos enfoques:

1. Análisis de citas web que extiende el análisis de citas tradicionales a la web.

2. Análisis de uso Web.

El análisis de uso evalúa el impacto científico a través de las estadísticas de lectores potenciales, por ejemplo, medición de las descargar y visitas de un artículo y su correlación con las citas recibidas por un documento. Los nuevos métodos cuantitativos de evaluación de investigación deben ser desarrollados y validados para extender y complementar los sistemas tradicionales de citas basados en el análisis bibliométrico, ya que estos sistemas obedecen a un tipo diferente de medición. Sin que de momento haya estudios concluyentes sobre si podrían ser de utilidad con fines de evaluación de la investigación. Por ello en 2013, NISO comenzó un ambicioso proyecto para desarrollar estándares y prácticas para altmetrics. Con la publicación del documento de Hodson (2014). En la primera fase del proyecto se celebraron tres reuniones y 30 entrevistas. El objetivo era recoger las aportaciones de todos los grupos interesados, resumir la discusión en este documento e identificar posibles puntos de acción para seguir trabajando en la Fase II del proyecto. Debido al formato abierto utilizado en las reuniones y entrevistas, se han recopilado una amplia gama de temas en torno a muchos aspectos de las métricas y evaluación, que también incluyen cuestiones que no están directamente relacionadas con las normas o mejores prácticas. Estas observaciones son una buena indicación de que una comunidad en evolución se preocupa por este tema y es factible que estos trabajos se consoliden en un trabajo productivo en el futuro. 
Altmetrics abarcan la actividad social en la forma de menciones en las redes sociales, la actividad académica en las bibliotecas digitales, índices de popularidad en los gestores de referencias, comentarios eruditos a través de blogs científicos y referencias en los medios sociales. Potencialmente, altmetrics podría en un futuro tener relación con la evaluación del profesorado y proceso de acreditación proporcionando a los comités de revisión información complementaria sobre la investigación a efectos sociales o interdisciplinares, y también podría ser considerado como herramienta para la concesión y dotación de financiación de proyectos de investigación. Ya que si los investigadores pueden demostrar que su investigación está generando una gran cantidad de interacción en la comunidad académica, tal información puede proporcionar una ventaja competitiva en un entorno de crisis financiera como el actual para la obtención de proyectos de investigación cuando aumentan los potenciales beneficiarios, pero no los recursos disponibles.

\section{Ventajas e inconvenientes de Almetrics}

Ventajas:

- Almetrics puede aplicarse a formatos no tradicionales como blog y presentaciones.

- Muestra el impacto en tiempo real.

- Muestra la atención recibida por una obra más allá de las citas.

En este mismo sentido NISO (Hodgson, 2014) considera que las ventajas de altmetrics sobre el Factor de Impacto son las siguientes:

1. Es abierto.

2. Es rápido.

3. Se basa en fuentes múltiples.

4. Es fácil de usar y comprender. 
Entre los argumentos en contra:

- Un alto número de menciones no es significativo de si la investigación es buena o mala. Miden la influencia social pero no la calidad de una investigación.

- Falta de consistencia y normalización entre los datos y sitios utilizados para la medición.

- El impacto social puede variar mucho respecto al impacto académico de una publicación.

- Algunas de estas herramientas de la web social son muy volátiles como el caso de Connotea que desapareció hace un par de años y era considerado como fuente por plataformas como altmetric.com

Una de las ventajas asociadas que conlleva este sistema de medición es que altmetrics recoge la influencia de la investigación desde el mismo momento de la publicación, es decir, que las evidencias de la influencia de esa investigación son inmediatas, desde el mismo día en que se edita el artículo tenemos constancia de las veces que se ha visualizado, descargado, compartido, twiteado, etc. en contraposición con la Bibliometría ortodoxa que necesita que se publiquen otros trabajos y que referencien una investigación para poder obtener las primeras evidencias de su impacto. Para ello son necesarios meses o años para generar la primera cita (índice de latencia), más en el caso de las ciencias sociales o humanidades. Almetrics desde el primer día en que aparece la publicación proporciona datos sobre cómo se usa una investigación.

La capacidad de citación entre disciplinas varía considerablemente, y además influyen en ella diferentes factores externos. Web of Knowledge de Thompson Reuters no tiene en cuenta todas las revistas, sólo las incluidas en el índice. Tampoco mide otros documentos asociados a la investigación, ni otros formatos como libros, presentaciones, set de datos que si son tenidos en cuenta por altmetrics.

La bibliometría tradicional no tiene en cuenta otros impactos, por ejemplo como ha sido utilizada esa publicación por parte del público, por instituciones, empresas, alumnos... Es 
decir, no tiene en cuenta el resto del ecosistema académico. Por ello algunas agencias como Wellcone Trust o Research Excellence Framework (REF) animan a los investigadores a aumentar su compromiso con el uso de otros indicadores alternativos que reflejen una más amplia influencia.

La comprensión de las diferencias en los recuentos obtenidos utilizando diferentes métodos es importante para el desarrollo de altmetrics. Recientemente se ha publicado el estudio How consistent are altmetrics providers? Study of 1000 PLOS ONE publications using the PLOS ALM, Mendeley and Altmetric.com APIs. para ello se recogió una muestra aleatoria de 30.000 DOls obtenidos de Crossref (15.000) y WoS (15.000), publicados en 2013. El resultado del estudio es que se encontraron importantes discrepancias e inconsistencias entre los proveedores de datos de métricas para los mismos conjuntos de datos. Es de destacar que los recuentos de lectores de Mendeley fueron muy similares entre los distintos agregadores, ya que los datos proceden directamente de Mendeley. Pero sin embargo los resultados para el recuento de tweets y comentarios de Facebook existen diferencias enormes respecto a la recopilación y notificación de estas métricas entre Altmetric.com y Lagotto, la aplicación de código abierto utilizada por Plos Almetrics.(«How consistent are altmetrics providers?», 2015).

Las posibles razones de inconsistencia son:

- Las diferencias en las métricas de informes (entre datos filtrados y datos en bruto / mensajes públicos y mensajes privados).

- Diferentes metodologías en las métricas de recolección y procesamiento (Twitter API).

- Diferentes actualizaciones: con posibles demoras en la recolección de datos o problemas de actualización. Utilización de diferentes identificadores (DOI, PMID, Identificación del arXiv) para las métricas de seguimiento.

- Dificultades en la especificación de la fecha de recogida de datos de influencia de la publicación (por ejemplo, diferentes fechas de publicación entre WoS y Crossref). 
- Los problemas de accesibilidad que difieren en diferentes plataformas de los editores (DOI resolver cuestiones URLs, problemas de cookies, denegación de acceso).

A este respecto NISO publicó una primera aproximación a la normalización de datos "Alternative Assessment Metrics (Altmetrics) Initiative"("Alternative Metrics Initiative National Information Standards Organization", 2016) con el objetivo de establecer las bases para una normalización del impacto social de la investigación. Otra iniciativa de normalización en el campo de la biomedicina es "Becker Library Research Model for Assesment Research Impact" un intento de poner de acuerdo a los sitios web, editores e investigadores para establecer un modelo riguroso de aplicación de estos indicadores para que sean adoptados por agencias, comités e investigadores de manera que enriquezcan el discurso académico.(«Alternative Metrics Initiative - National Information Standards Organization», 2016).

Durante los últimos años algunas plataformas y editores como Plos o Elsevier han adaptado a sus bases de datos a las medidas altmétricas. Esto también tiene que ver mostrarse innovadoras y con la adopción del modelo de acceso abierto denominado " $e l$ autor paga" con la finalidad de ofrecer un valor añadido a este modelo de negocio.

Almetrics ofrece un instantáneo y fácil acceso al impacto inmediato de una investigación en tiempo real, desde el primer momento de la publicación ofrece datos de visualizaciones, descargas, veces que se ha compartido o comentado en redes sociales, etc. Lo que proporciona al autor indicios de como se está promocionando su trabajo, y a su vez añade un valor al esfuerzo del editor en la consecución de esta tarea.

A pesar del nombre, Altmetrics complementa, no sustituye a la bibliometría. Es un suplemento a la bibliometria proporcionando una imagen más amplia del impacto de una publicación. Facilitando de esta manera datos sobre su impacto antes de que se produzca la primera cita. (Torres, Cabezas y Jiménez, 2013) En plataformas como Nature o Plos podemos ver esta integración del sistema tradicional de citas e indicadores altmétricos. Además también contribuye a gestionar y promocionar la identidad digital del investigador, ya que muestra en tiempo real la capacidad de influencia en los nuevos 
medios. (Williams y Padula, 2015).

\section{Plataformas y herramientas de medición almétrica}

\section{Altmetric.com}

Altmetric.com es una herramienta que se utiliza bajo suscripción, muestra el impacto de la investigación a sus autores y lectores de modo muy gráfico y nuevo. Monitorear, buscar y medir todas las conversaciones acerca de los artículos de una revista, así como los publicados por sus competidores. Recoge las menciones de artículos académicos de todas partes de la Web mediante la recopilación de menciones en los periódicos, blogs, redes sociales y otros sitios web. En cuestión de minutos, permite al autor disponer de los datos altmétricos para mostrarlos en su plataforma o aplicación. El algoritmo Altmetric.com calcula una puntuación global teniendo en cuenta el volumen, la fuente y el autor en función de las menciones que recibe un documento. Esto incluye las menciones de artículos académicos en los sitios de medios sociales. (por ejemplo, Twitter, Facebook, Pinterest, Google+), blogs científicos, muchos medios de comunicación convencionales, incluyendo The New York Times, The Guardian, las publicaciones en idiomas distintos al inglés, como Die Zeit y Le Monde y publicaciones de interés especial como Scientific American y New Scientist, sitios de revisión por pares de referencia como Publons.

Recoge las menciones de artículos académicos de todas partes de la Web mediante la recopilación de menciones en los periódicos, blogs, redes sociales y otros sitios web. En cuestión de minutos, permite al autor disponer de los datos Altmetrics para insertarlos y mostrarlos en su plataforma o aplicación. Hoy en día la base de datos de Altmetric contiene menciones de más de 4 millones de fuentes de investigación (incluyendo artículos de revistas, bases de datos, imágenes, documentos, informes y más), y está en constante crecimiento. Para realizar un seguimiento de las menciones en línea para una parte específica de la investigación, se tiene en cuenta tres cuestiones:

- Un documento fuente (artículo de revista, informes, imágenes) 
- Un identificador (DOI, RePeC, PUbMed id, Arxiv ID, SSRN...)

- Menciones recibidas.

Los identificadores también ayudan a reconocer las diferentes versiones de una investigación y a su desambiguación. Por ejemplo, un artículo de una revista puede estar originalmente disponible en una plataforma editorial y con un DOI, y luego estar alojado en PubMed o en un repositorio institucional.

La cantidad de cada color en el donut cambiará dependiendo de qué fuentes ha recibido la atención, los resultados de una salida de la investigación. En la siguiente figura vemos que cuando la atención ha sido recibida por medios de comunicación tiene fundamentalmente colores rojos, cuando las menciones vienen de blog tendrá un color predominantemente amarillo, y en el tercer donut la atención fundamental proviene de twitter.

La puntuación Altmetric es calculada automáticamente por conteo ponderado en función de la atención recibida por la investigación. Se basa en 3 factores principales:

- Volumen. Número de menciones.

- Fuente. Cada categoría es puntuada de diferente forma. Por ejemplo puntúa más la mención en un artículo de prensa que en un blog (ver tabla).

- Autores. Puntúa en función de la categoría del autor que haya hecho la medición, o en que revista se haya hecho en función de su impacto o audiencia. 


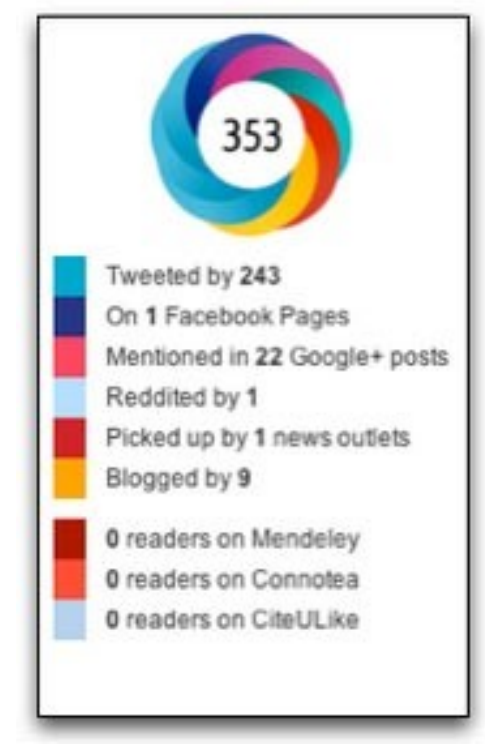

Fig. 5. Rosco almetric.com

El algoritmo también mide factores de carácter de autoridad de los autores, por lo que una mención realizada por un experto en el campo vale más que una mención hecha por un estudiante. La representación visual - la rosquilla Altmetric.com - muestra la distribución proporcional de menciones por tipo de fuente. Cada tipo de fuente presenta un color diferente - azul para Twitter, amarillo para los blogs, y el rojo para Mendeley y otros tonos de rojo para los principales medios de comunicación. Los enlaces a las fuentes de datos también están disponibles. Altmetric.com recoge alrededor de cien mil menciones a la semana, con cerca de 3.000 artículos nuevos incluidos cada día.7.

En conjunto, el resultado final de la puntuación representa una aproximación ponderada de todas las menciones que se han hecho en diferentes medios a esa investigación. La puntuación para los artículos de Wikipedia es estática. Esto significa que si una investigación se ha mencionado en Wikipedia, la puntuación se incrementará en 3 puntos. El redondeo siempre es con números enteros. En el caso de Twitter, los retweets se puntúan con 0.85 puntos, en lugar de 1, ya que reciben una mención de segunda mano en lugar de la atención inicial. El total combinado de estos re-tweets siempre se redondea al número entero más próximo. En el caso de noticias en medios de comunicación se les asigna un nivel, basado en el alcance que tiene ese medio. la puntuación por lo tanto depende del nivel de esa fuente de la noticia. Esto significa que una mención de un medio de comunicación nacional y popular, como The New York 
Times contribuirá más a la puntuación que una mención noticias de una publicación de nicho más pequeño.

La ficha resumen proporciona los datos demográficos de los usuarios y la "puntuación en contexto" y cada pestaña muestra el nivel de atención que la investigación ha recibido y se compara con otros productos publicados en la misma época, o en la misma revista.

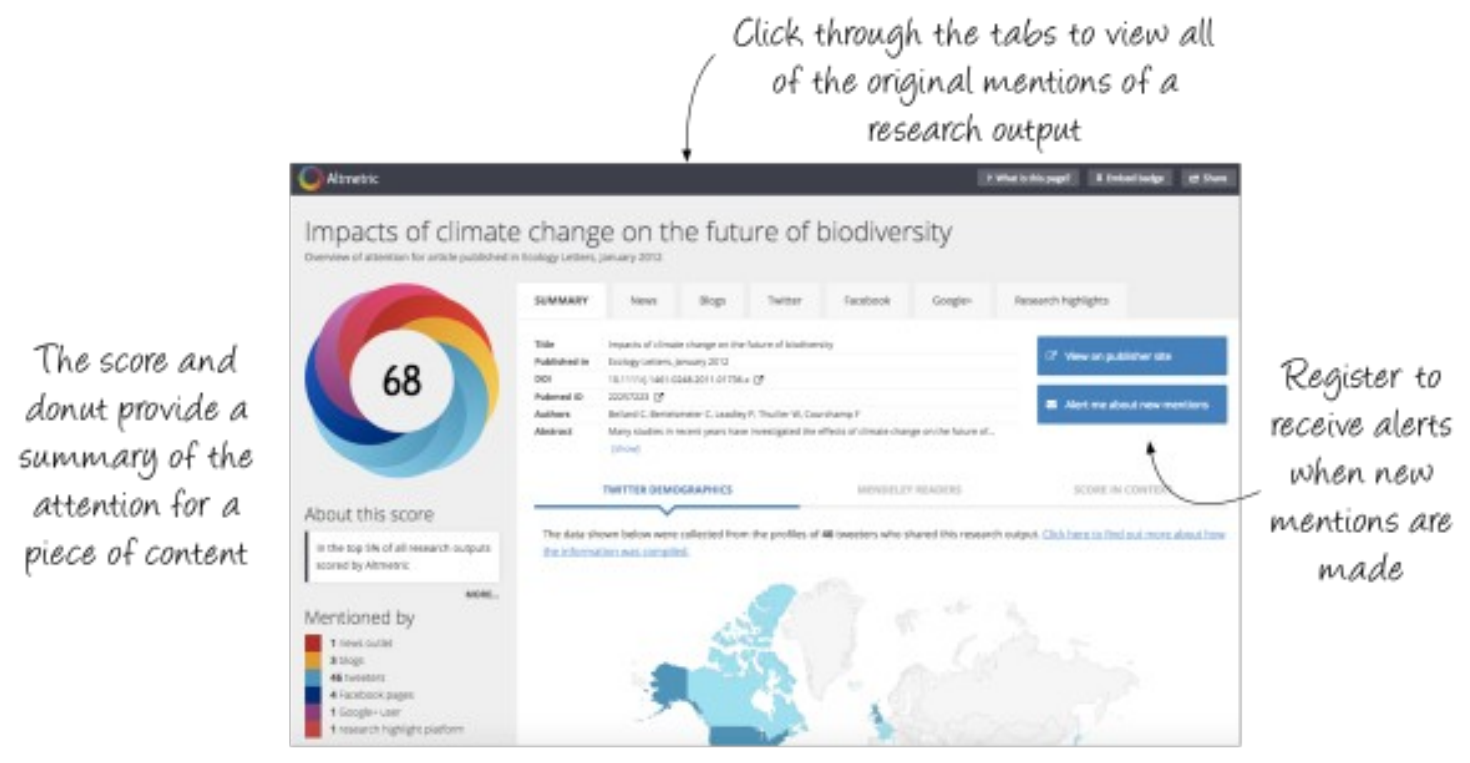

Fig. 6. Ficha resumen altmetric.com

\section{Plum Analytics}

Plum (que significa ciruela) es el portal de métricas de investigación de Ebsco. Realiza un seguimiento de todos los productos de la investigación en cualquier forma, proporcionando una poderosa herramienta que aumenta la capacidad de la métrica tradicional. La herramienta PlumX reúne a las métricas a través de cinco categorías: citaciones, uso, menciones, capturas recogidas de los datos de proporcionados por los medios sociales. Los documentos incluyen: artículos, entradas del blog, capítulos de libros, libros, casos, ensayos clínicos, comunicaciones a congresos, conjuntos de datos, cifras, subvenciones, entrevistas, cartas, medios de comunicación, patentes, posters, presentaciones, código fuente, tesis / disertaciones, vídeos, páginas web. Plum 
proporciona datos objetivos sin establecer ponderaciones o ranking como altmetric.com. También es posible añadir widgets de PlumX a su repositorio institucional, perfiles de investigadores, sitios web del departamento, o blogs, etc.

\section{Impact Story}

A cambio de una cuota mensual se pueden tener un perfil de ImpactStory, con un período de prueba gratuita inicial, que permite al investigador construir un CV en línea que proporciona datos sobre la atención recibida de sus trabajos en los medios sociales

\section{PLOS metrics}

Las métricas PLOS están libremente disponible directamente desde las páginas de artículos publicados en PLoS.

\section{Uso de Almetrics}

Alguna de las grandes multinacionales de la investigación científica como Elsevier, que ha sido durante mucho tiempo un defensor a ultranza de la bibliometría ortodoxa basada únicamente en las citas que recibe un artículo, está interesándose en la comprensión de cómo se pueden utilizar estas nuevas medidas en relación con los datos de uso y citación, para proporcionar nuevos indicadores significativos para la comunidad investigadora. Elsevier ha empezado a participar en el proyecto NISO Altmetrics y esta línea sigue su proyecto Article Usage Reports, un programa que informa a autores que han publicado en las revistas de Science Direct y Elsevier.com sobre cómo se está visualizando su artículo. También Elsevier ha establecido relaciones con Altmetric.com, y además se ha unido a un proyecto piloto a través de Kudos para investigar cómo los autores de Elsevier pueden publicitar sus investigaciones. 

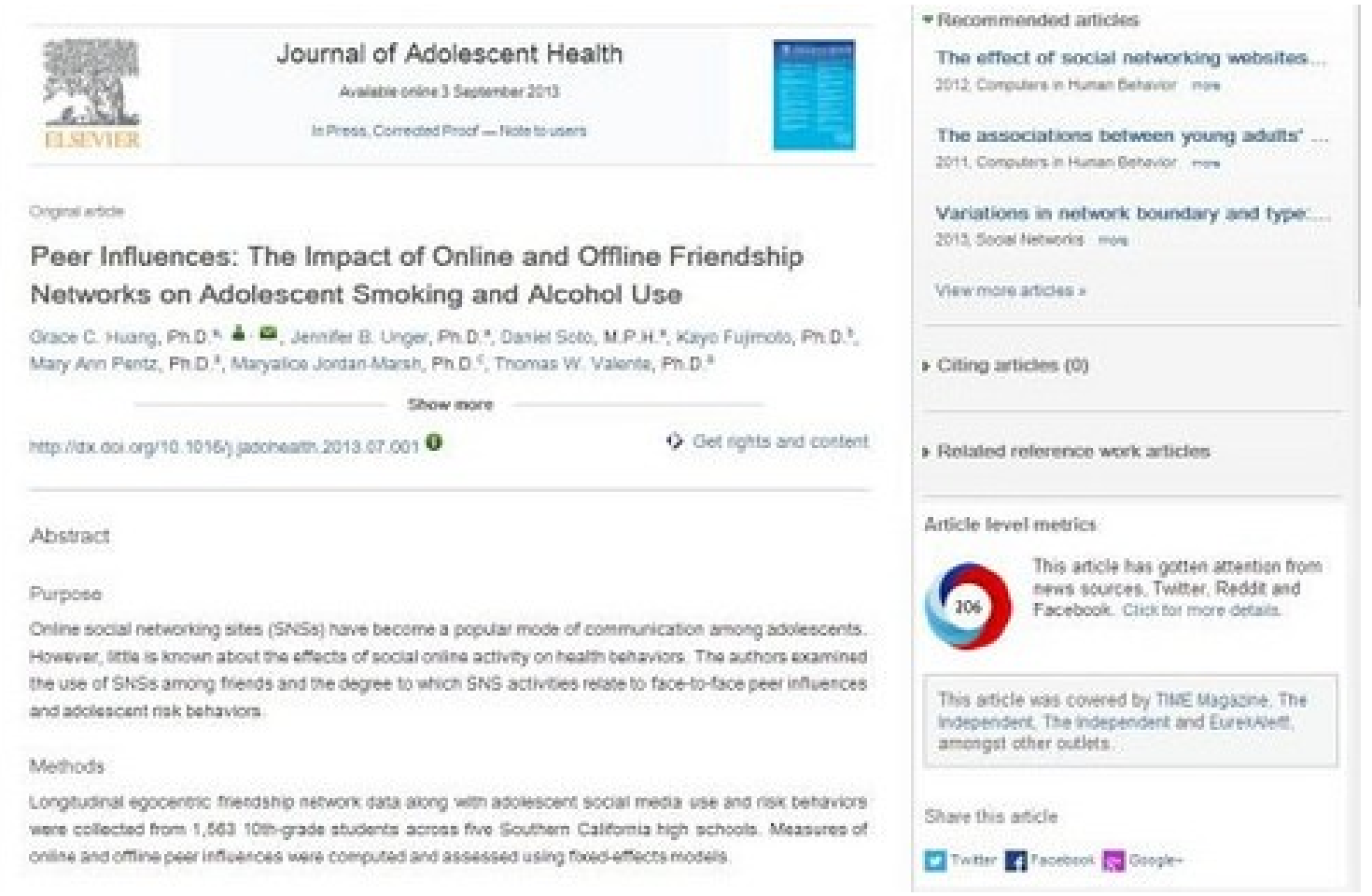

Fig. 7. Página de Elsevier con el donut altmetric.com

Editores como Nature o Science reconocen que el uso de altmetrics les ha proporcionado una mayor cobertura de sus publicaciones en los medios y eventualmente un mayor número de citas. Canales nicho como blog o revistas de poca cobertura también reconocen que el uso de medidas altmétricas beneficia un mayor reconocimiento y visibilidad, construyendo estrategias más eficaces para la promoción de sus publicaciones. En esta misma línea Elsevier reconoce que aquellos artículos donde ha incluido medidas altmétricas reciben mayor atención. Taylor \& Francis también incluye datos almétricos en su Top 20. MIT Press también está incorporando medidas altmétricas en sus publicaciones de Batches Books Series. Los departamentos de marketing y publicación y publicación utilizan estas medidas para poder tener datos fiables y determinar el éxito de sus páginas, conocer la procedencia geográfica de uso y de esta manera establecer estrategias de mercado.

En instituciones como Michigan Publishing almetrics contribuye a disponer de información sobre las publicaciones en tiempo real para demostrar el valor de la actividad editorial y de esta manera justificar la inversión llevada a cabo por la entidad pública. Por ello también ha incorporado medidas almétricas a su programa de 
publicación de libros en acceso abierto "Digital Culture Books" y al repositorio institucional "Deep Blue". Esto está siendo especialmente útil para los libros que no disponían de Factor de Impacto, y de este modo tienen información sobre su uso. Teniendo de esta manera evidencias que puedan justificar la inversión llevada a cabo ante los organismos de financiación.

Almetrics es especialmente atractivo para aquellas disciplinas y áreas geográficas que tradicionalmente han sido excluidas del análisis bibliométrico, ya que no están suficientemente representadas en bases de datos como Web of Science (WoS) o Scopus, como las ciencias sociales y las humanidades. Según la investigación "Do "altmetrics" correlate with citations? Extensive comparison of altmetric indicators with citations from a multidisciplinary perspective." que hace un análisis de la presencia de diferentes indicadores altmétricos proporcionados por Altmetric.com en distintos campos científicos, centrándose especialmente en su relación con las citas. Los resultados confirman que la presencia y densidad de los recuentos altmétricos siguen siendo muy bajas, entre sólo un $15 \%$ a un $24 \%$ de publicaciones presentan alguna actividad altmétrica concentrada en las publicaciones más recientes, aunque su presencia ha aumentado con el tiempo. Las publicaciones de las ciencias sociales, humanidades y las ciencias médicas y de la vida muestran la más alta presencia de indicadores altmétricos, indicando su valor potencial y el interés para estos campos. El análisis de las relaciones entre altmetrics y citas confirma afirmaciones anteriores de correlaciones positivas pero relativamente débiles, lo que apoya la idea de que altmetrics no reflejan el mismo tipo de impacto que las citas. (Costas, Zahedi y Wouters, 2015).

Por todo ello es importante formar al investigador sobre la capacidad de estos medios como mecanismos de tracción y visibilidad a su investigación reforzando su identidad digital. Una buena herramienta en este sentido es la publicación "Users, narcissism and control - tracking the impact of scholarly publications in the 21st century (Wouters $y$ Costas, 2012) un informe que analiza la explosión de las herramientas de seguimiento que han acompañado el surgimiento de instrumentos de información basados en la web. El informe recomienda iniciar un programa de investigación concertado en la dinámica, propiedades y uso potencial de las métricas web basadas en los nuevos sistemas de 
medición del impacto de la publicación. Su objetivo es contribuir al desarrollo de herramientas más útiles para la comunidad científica y académica. Este programa debe abordar por lo menos las siguientes herramientas: F1000, Microsoft Academic Research, Total-Impact, PlosONE altmetrics, y Google Scholar. El programa además desarrolla los siguientes temas: los conceptos clave de la investigación de nuevas métricas web y altmetrics, la estandarización de herramientas y datos, y el uso de las nuevas métricas. Algunos autores hablan de la función de estas herramientas como una forma de revisión por pares tras la publicación (Post-Publication Peer Review); además de proporcionar fuentes de datos y citas de otros materiales como presentaciones, cursos, artículos de blogs de los que habitualmente no se tenían datos (Torres-Salinas, 2012).

\section{Conclusiones}

El surgimiento del movimiento de acceso abierto y los avances en los flujos de trabajo de la publicación digital están aportando más oportunidades que nunca para incrementar el impacto de la investigación tanto dentro como fuera del contexto académico. Unido a ello están los avances en el análisis de los 'big data' cada vez más interconectados, por lo que en la actualidad se incrementan las maneras para medir el impacto de la investigación en una forma más extensa que como se ha venido realizando en los últimos 50 años. Como la investigación se mueve en línea y las referencias de la de investigación divergen más allá de los confines de las citas bibliométricas, muchos investigadores y editores de revistas han comenzado a buscar nuevos indicadores de impacto para captar mejor estos comportamientos cambiantes. Altmetrics han surgido como un indicador alternativo que puede ofrecer a investigadores y editores de revistas una visión más holística del alcance y la utilidad de sus publicaciones. (Williams \& Padula, 2015).

El impacto de estos cambios en la comunicación científica y en la credibilidad de la información todavía está por verse, pero es evidente que los medios sociales han encontrado una importante tracción en casi todos los sectores de la educación y como consecuencia de ello están generando una influencia en los procesos de comunicación científica y en los hábitos y comportamientos de los investigadores de todas las 
disciplinas.

Estas nuevas posibilidades de comunicación también ofrecen nuevas oportunidades para la formación, el análisis y evaluación de la investigación. Los científicos y los investigadores están utilizando de forma rutinaria las aplicaciones basadas en web en sus investigaciones. Los investigadores incorporan cada vez más herramientas para mejorar el impacto de su investigación (González-Fernández-Villavicencio et al., 2015). Es en este nivel donde la biblioteca juega un papel indispensable en la formación de competencias, destrezas y habilidades informativas que repercutirá en la valoración social del profesional, su satisfacción laboral y en última instancia en la calidad de la propia institución. Se destacan los aspectos más sobresalientes en los nuevos paradigmas de comunicación y difusión científica y se recomiendan las acciones de formación más adecuadas al respecto.

Bibliotecas y bibliotecarios poseen la cualificación necesaria para proporcionar en su instrucción el uso apropiado de altmetrics para promover a la comunidad académica global. El impacto y el valor de la investigación producida en sus instituciones De acuerdo con un informe sobre NISO altmetrics, la gran cantidad de resultados de Google que citan tanto "Libguides" y "altmetrics" "indican que las bibliotecas ya están incorporando información altmétrica en los recursos para la comunicación científica, el impacto y la gestión de citas," pero el informe llega a la conclusión de que "la eficacia de estas guías sigue siendo desconocida.

Altmetrics, la medida de la actividad académica en las redes sociales y en las herramientas sociales, es un término de moda importante en este momento, y aunque es una disciplina muy nueva, su interés está creciendo rápidamente. Altmetrics ofrecen una visión alternativa sobre el uso e impacto de la investigación sobre quienes leen artículos académicos, y esta información ha impulsado a autores, investigadores, editores a tratar de entender cuál es la importancia de estos datos (Torres, Cabezas y Jiménez January, 2013).

Pero para que esto sea una realidad es muy importante entender las similitudes 
potenciales o diferencia en las cifras a través de diferentes agregadores altmetrics. Рara ello es necesario recurrir a las mejores prácticas en la recopilación de datos altmetric tanto por parte de los proveedores altmetric como de los agregadores y editores. Para ello es conveniente desarrollar normas, directrices y recomendaciones para introducir transparencia y coherencia a través de proveedores y agregadores.

El valor de altmetrics como una herramienta complementaria de análisis de citas es evidente, aunque se sugiere que se debe investigar más profundamente sobre esta cuestión para desentrañar el significado y el valor de los indicadores para la evaluación de su potencial.

\section{Bibliografía}

AGUILLO, I., 2011. Is Google Scholar useful for bibliometrics? A webometrics analysis. Scientometrics [en línea], Disponible en: http://link.springer.com/article/10.1007\%2Fs11192-011-0582-8.

Alternative Metrics Initiative - National Information Standards Organization. [en línea], 2016. [Consulta: 24 marzo 2016]. Disponible en: http://www.niso.org/topics/tl/altmetrics_initiative/.

BURGELMAN, J.-C., OSIMO, D. y BOGDANOWICZ, M., 2010. Science 2.0 (change will happen....). First Monday [en línea], vol. 15, no. 7. [Consulta: 29 marzo 2016]. ISSN 13960466. Disponible en: http://firstmonday.org/ojs/index.php/fm/article/view/2961.

COSTAS, R., ZAHEDI, Z. y WOUTERS, P., 2015. Do altmetrics correlate with citations? Extensive comparison of altmetric indicators with citations from a multidisciplinary perspective. En: arXiv: 1401.4321, Journal of the Association for Information Science and Technology [en línea], vol. 66, no. 10, pp. 2003-2019. [Consulta: 28 marzo 2016]. ISSN 23301635. DOI 10.1002/asi.23309. Disponible en: http://arxiv.org/abs/1401.4321.

DAS, A.K., 2015. Research evaluation metrics [en línea]. S.l.: UNESCO Publishing. [Consulta: 28 marzo 2016]. Disponible en: https://books.google.es/books? $\mathrm{hl}=$ es\&lr=\&id=MZlYCgAAQBAJ\&oi=fnd\&pg=PA1\&dq=Research+evaluation+metrics\&ots= 
1HEGFJOn1r\&sig=ywzIZsqD1MPskjP3IAZai4oXSSA.

GONZÁLEZ-FERNÁNDEZ-VILLAVICENCIO, N., DOMÍNGUEZ-AROCA, M.-I., CALDERÓNREHECHO, A. y GARCÍA-HERNÁNDEZ, P., 2015. ¿Qué papel juegan los bibliotecarios en las altmetrics? Anales de Documentación. Anales de Documentación [en línea], vol. 18, no. 2, pp. 1-19. Disponible en: http://eprints.rclis.org/25480/1/222641-813251-1-PB $\% 20 \% 281 \% 29 . p d f$.

HODGSON, C., 2014. NISO Altmetrics Standards Project White Paper [en línea]. S.l.: Altmetrics Steering Group. Disponible en: http://www.niso.org/apps/group_public/download.php/13295/niso_altmetrics_white_pa per_draft_v4.pdf.

How consistent are altmetrics providers? Study of 1000 PLOS ONE publications using the PLOS ALM, Mendeley and Altmetric. com APIs. [en línea], 2015. [Consulta: 28 marzo 2016].

Disponible

en:

http://www.academia.edu/11617808/How_consistent_are_altmetrics_providers_Study_ of_1000_PLOS_ONE_publications_using_the_PLOS_ALM_Mendeley_and_Altmetric._com APIs.

INNOVATIONS, 101, 2015. First 1000 responses - most popular tools per research activity. Innovations in Scholarly Communication [en línea]. [Consulta: 29 marzo 2016]. Disponible en: https://101innovations.wordpress.com/2015/06/23/first-1000-responsesmost-popular-tools-per-research-activity/.

NEYLON, C., 2011. Re-use as Impact: How re-assessing what we mean by «impact» can support improving the return on public investment, develop open research practice, and widen engagement. Altmetrics [en línea], Disponible en: http://altmetrics.org/workshop2011/neylon-v0/.

PRIEM, J., PIWOWAR, H.A. y HEMMINGER, B.H., 2011. Altmetrics in the wild: An exploratory study of impact metrics based on social media. Metrics 2011: Symposium on Informetric and Scientometric Research. New Orleans, LA, USA [en línea], Disponible en: http://jasonpriem.org/self-archived/PLoS-altmetrics-sigmetrics11-abstract.pdf. 
PRIEM, J., TARABORELLI, D. y GROTH, P., 2010. Alt-metrics: a manifesto. Alt-metrics [en línea], Disponible en: http://altmetrics.org/manifesto/.

SCIENCE, I.C. of, 2014. Open access to scientific data and literature and the assessmentof research by metrics [en línea]. S.l.: International Council of Science. Disponible en: http://www.icsu.org/general-assembly/news/ICSU\%20Report\%20on\%200pen \%20Access.pdf.

THELWALL, M., HAUSTEIN, S., LARIVIÈRE, V. y SUGIMOTO, C.R., 2013. Do Altmetrics Work? Twitter and Ten Other Social Web Services. PLoS ONE [en línea], vol. 8, no. 5. ISSN 19326203. Disponible en: http://dx.doi.org/10.1371/journal.pone.0064841.

TORRES, D., CABEZAS, Á. y JIMÉNEZ, E., Altmetrics: nuevos indicadores para la comunicación científica en la Web 2.0. Altmetrics: New Indicators for Scientific Communication in Web 2.0. Revista Comunicar [en línea], vol. 21, no. 41, pp. 53-60. ISSN 1134-3478. DOI 10.3916/C41-2013-05. Disponible en: http://dx.doi.org/10.3916/C412013-05.

WILLIAMS, C. y PADULA, D., 2015. The Evolution of Impact Indicators: From bibliometrics to altmetrics [en línea]. S.l.: Almetric.com Scholastica. Disponible en: http://scholasticahq.com/altmetrics-the-evolution-of-impact-indicators http://docs.scholastica.s3.amazonaws.com/altmetrics/evolution-of-impactindicators.pdf.

WOUTERS, P. y COSTAS, R., 2012. Users, narcissism and control - tracking the impact of scholarly publications in the 21st century [en línea]. S.l.: SURFfoundation. Disponible en: http://www.surffoundation.nl/nl/publicaties/Documents/Users\%20narcissism\%20and \%20control.pdf. 